\title{
The Shapley Value: Its Use and Implications on Internet Economics
}

\author{
Richard T.B. Ma \\ Columbia University \\ tbma@ee.columbia.edu
}

\author{
John C.S. Lui, Dah-Ming Chiu \\ The Chinese University of Hong Kong \\ \{cslui@cse,dmchiu@ie\}.cuhk.edu.hk
}

\author{
Vishal Misra, Dan Rubenstein \\ Columbia University \\ \{misra,danr\}@cs.columbia.edu
}

\section{Introduction}

The Internet is composed of thousand of autonomous Internet Service Providers (ISPs). On the one hand, they cooperate with one another to provide services for their customers; on the other hand, they compete with each other by using selfish routing and interconnecting strategies to maximize their own profits. Currently, ISPs use bilateral settlements to decide the financial compensation one ISP pays to another. However, without an appropriate settlement model, ISPs disputes might lead to disgraceful consequences. For example, Level 3 unilaterally terminated its "settlement free" peering relationship with Cogent on October 5, 2005. This disruption resulted in at least $15 \%$ of the Internet to be unreachable for the users who utilized either Level 3 or Cogent for Internet access. Although both companies restored peering connections several days later with a new on-going negotiation, Level 3's move against Cogent exhibited an escalation of the tension that necessitates a new settlement for ISPs.

In this paper, we introduce a cooperative game solution, namely the Shapley value [1], [2], and summarize the results of a series of papers [3], [4] that apply the Shapley value to address the ISP settlement problems. We believe that it provides a new angle for ISPs to negotiate cooperations, settle disputes, and create better services for customers.

\section{The Shapley Value}

We start introducing the Shapley value as a profit-sharing solution in a general cooperative environment. We define $\mathcal{N}$ as a set of cooperative players and $v$ as a profit function defined on any subset $\mathcal{S} \subseteq \mathcal{N} . v(\mathcal{S})$ defines the profit generated by the set $\mathcal{S}$ independently. We define $\varphi=\left\{\varphi_{1}, \varphi_{2}, \cdots, \varphi_{|\mathcal{N}|}\right\}$ as a profit-sharing function, where each $\varphi_{i}$ defines the profit distributed to $i \in \mathcal{N}$. Based on the profit function $v$, we want to find an appropriate profit sharing function $\varphi$ for all players $\mathcal{N}$ to share profit. By appropriateness, we require the profitsharing function $\varphi$ to satisfy multiple desirable properties.

Property 1 (Efficiency): $\sum_{i \in \mathcal{N}} \varphi_{i}(\mathcal{N}, v)=v(\mathcal{N})$.

Property 2 (Symmetry): If $v(\mathcal{S} \cup\{i\})=v(\mathcal{S} \cup\{j\})$ for all $\mathcal{S} \in \mathcal{N} \backslash\{i, j\}$, then $\varphi_{i}(\mathcal{N}, v)=\varphi_{j}(\mathcal{N}, v)$.

The efficiency property requires that the assigned profit balances the profit generated by the set of players. In other words, the profit-sharing solution does not contribute or reduce extra profit. The symmetry property addresses a fairness issue which requires that if two players contribute the same, they should receive the same amount of profit.

We consider a scenario with two players $\mathcal{N}=\{1,2\}$ and $v(\{1\})=v(\{2\})=0, v(\{1,2\})=V$. This scenario models a situation where each individual player cannot generate profit, but both of them together can generate profit of $V$. Intuitively, both players should evenly share the profit, i.e. $\varphi_{1}=\varphi_{2}=$ $\frac{1}{2} V$. This solution satisfies the above two properties. However, for more complicated scenarios, the above two properties do not uniquely determine a profit-sharing solution. We further consider a scenario with three players $\mathcal{N}=\{1,2,3\}$ and $v(\{1\})=v(\{2\})=v(\{3\})=0, v(\{1,2\})=v(\{1,3\})=$ $v(\{1,2,3\})=V$. We can imagine a simplified PC market in Figure 1 with player 1 to be the operating system producer and player 2 and 3 to be two microprocessor producers. Suppose the total profit generated from the PC market is $V$. This profit can be generated only if we have the operating system and at least one of the microprocessor to cooperate. To satisfy the above two properties, we require the profit-sharing solution to satisfy the following two equations: $\varphi_{1}+\varphi_{2}+\varphi_{3}=V$ and $\varphi_{2}=\varphi_{3}$. However, these two equations do not uniquely determine a profit-sharing solution. We introduce one more desirable property that balances the mutual benefits the players contribute to one another.

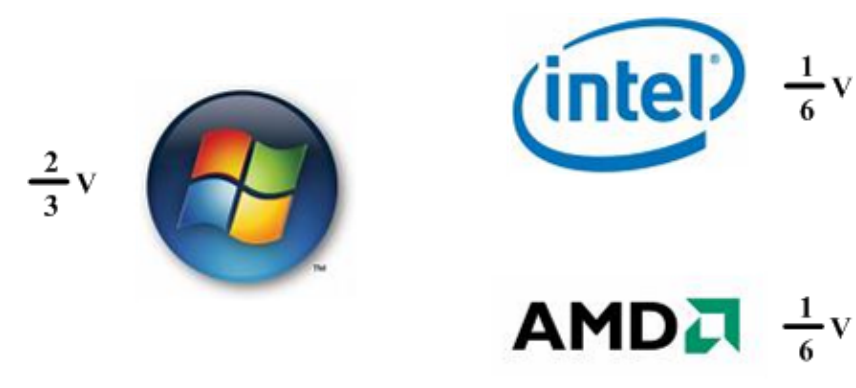

Fig. 1. A PC market profit-sharing example.

Property 3 (Balanced Contribution): For any $i, j \in \mathcal{N}, j$ 's contribution to $i$ equals $i$ 's contribution to $j$, i.e. $\varphi_{i}(\mathcal{N}, v)-$ $\varphi_{i}(\mathcal{N} \backslash\{j\}, v)=\varphi_{j}(\mathcal{N}, v)-\varphi_{j}(\mathcal{N} \backslash\{i\}, v)$.

Suppose we pick up a pair of players 1 and 2, the balanced contribution property requires:

$$
\varphi_{1}(\mathcal{N}, v)-\varphi_{1}(\mathcal{N} \backslash\{2\}, v)=\varphi_{2}(\mathcal{N}, v)-\varphi_{2}(\mathcal{N} \backslash\{1\}, v),
$$


where $\varphi_{2}(\mathcal{N} \backslash\{1\}, v)=0$ and $\varphi_{1}(\mathcal{N} \backslash\{2\}, v)=\frac{1}{2} V$. This is because without player 1 , no profit can be generated and without player 2 , both players should be evenly share the total profit. After solving the three equations, we obtain $\varphi_{1}=\frac{2}{3}$ and $\varphi_{2}=\varphi_{3}=\frac{1}{6}$ as shown in Figure 1.

Myerson [5] showed that there exists a unique function $\varphi$ satisfying Efficiency, Symmetry and Balanced Contribution properties. This unique function is the Shapley value proposed by Llyod Shapley [1] in his original paper published in 1953.

Definition 1: The marginal contribution of player $i$ to a set $\mathcal{S} \subseteq \mathcal{N} \backslash\{i\}$ is defined as $\Delta_{i}(v, \mathcal{S})=v(\mathcal{S} \cup\{i\})-v(\mathcal{S})$.

Definition 2: The Shapley value $\varphi$ is defined by

$$
\varphi_{i}(\mathcal{N}, v)=\frac{1}{|\mathcal{N}| !} \sum_{\pi \in \Pi} \Delta_{i}(v, S(\pi, i)) \quad \forall i \in \mathcal{N},
$$

where $\Pi$ is the set of all $|\mathcal{N}|$ ! orderings of $\mathcal{N}$ and $S(\pi, i)$ is the set of players preceding $i$ in the ordering $\pi$.

The Shapley value of a player can be interpreted as the expected marginal contribution $\Delta_{i}(v, \mathcal{S})$ where $\mathcal{S}$ is the set of players preceding $i$ in a uniformly distributed random ordering. The Shapley value depends only on the values $\{v(\mathcal{S}): \mathcal{S} \subseteq \mathcal{N}\}$. The Shapley value also satisfies a bunch of additional desirable properties, e.g. additivity, dummy, strong monotonicity, consistent. Eyal Winter's survey [2] provides detail discussions about Shapley value's properties and applications.

\section{ISP Profit Sharing}

Internet Service Providers (ISPs) cannot operate independently. They require the cooperation of other ISPs to provide Internet services to their customers. Due to this cooperative nature, we consider the Shapley value as an appropriate solution for ISPs to share profit. In [4], we adopted the contenteyeball model by Faratin et al. [6] and considered a two-sided market model as shown in Figure 2. On the left side, content

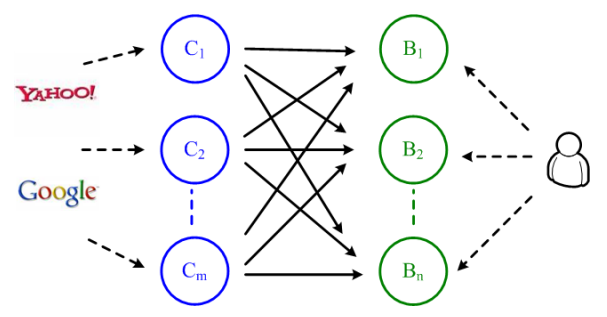

Fig. 2. Shapley profit-sharing for eyeball and content ISPs.

providers, e.g. Google and Yahoo, provide information through $m$ content ISPs denoted as $C_{i}$; on the right side, end-customers access Internet and download information from $n$ eyeball ISPs denoted as $B_{j}$. Suppose the total profit generated by serving both content providers and end customers is $V$. We derived the Shapley value profit-sharing solution for the ISPs as follows.

$$
\varphi_{C_{i}}=\frac{n}{m(m+n)} V, \quad \varphi_{B_{j}}=\frac{m}{n(m+n)} V .
$$

Besides the three desirable properties we discussed in the previous section, we make the following observations:
- Each ISP's Shapley profit is proportional to the number of ISPs of the other kind.

- Each ISP's Shapley profit is inversely proportional to the number of ISPs of the same kind.

Intuitively, when the number of same type of ISPs increases, these similar ISPs make substitution for each other and therefore, the other type of ISPs can make higher leverage to share higher profit. In [4], we also discussed inelastic customer demands from different geographic regions, where the Shapley value profit can be decomposed linearly by the additive property of the Shapley value.

\section{ISP Routing and Interconnecting Incentives}

Although ISPs have to cooperate with each other to provide Internet services, they are selfish business entities whose major objective is to maximize their own profits. ISPs often make selfish routing, e.g. hot-potato routing, and interconnecting, e.g. peering, decisions. Without an appropriate compensation structure, these individual selfish behaviors make the Internet converged into an inefficient system. In [3], we enforce a Shapley value mechanism, which distributes the profit to ISPs according to the Shapley value, and explore the selfish

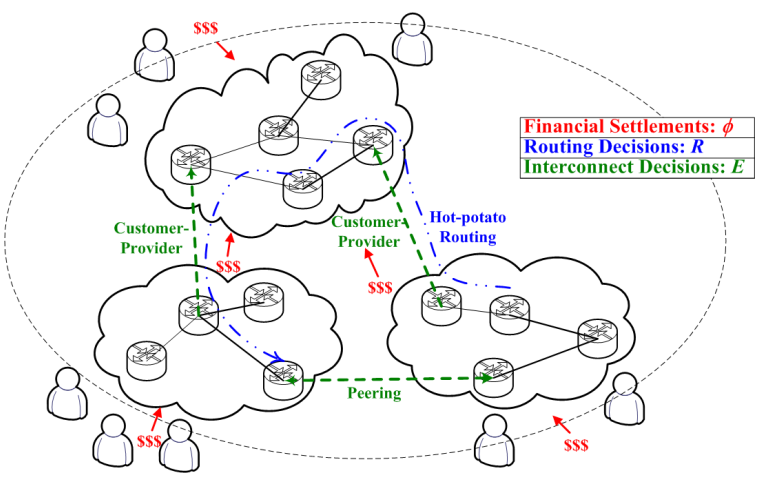

Fig. 3. A view of ISP interactions of the Internet.

behaviors of individual ISPs as well as the global equilibrium of the system under the enforcement of the Shapley value profit-sharing. Figure 3 illustrates our view of a three-layer framework of ISP interactions. On the top, we see that ISPs make financial settlements. Based on these financial settlements, each ISP makes underlying routing and interconnecting decisions to maximize their profits. In particular, we explore the selfish behaviors of ISPs under the Shapley value settlement. The profit function under this context is:

$$
v(\mathcal{S})=\text { Revenue }- \text { Routing Cost. }
$$

Notice that each ISP's selfish behavior affects the profit of a group of ISPs: routing decision affects routing costs and interconnecting decision affects both revenue sources and routing costs. We derived the following results when ISPs are encouraged by the Shapley value settlement.

- Given any fixed interconnecting topology, each ISP's optimal routing strategy maximizes global profit.

- Any routing Nash equilibrium maximizes global profit. 
- With negligible interconnecting cost, ISPs can obtain non-decreasing profits by interconnecting with other ISPs.

These results imply that if we can implement the Shapley value settlement among ISPs, selfish local decisions will coincide with global optimal decisions. Therefore, hot-potato routing and de-peering behaviors will not be rational for ISPs to perform.

\section{Future Work and Conclusion}

We have exhibited the Shapley value as a profit-sharing solution for ISPs under simple scenarios as well as ISPs' selfish behaviors under the Shapley value settlement. Both the desirable properties and the incentives provided by the Shapley value make it an ideal settlement solution for ISPs. However, how to implement the Shapley value solution in practice is still an open question. Two of the major difficulties are a) the calculation of the Shapley value, i.e. Equation (1), is computationally expensive and does not always have a simple form like Equation (2), and b) the Shapley value settlement is a multi-lateral agreement which is more difficult to implement than the currently implemented bilateral agreements between pairs of ISPs. To tackle these difficulties, we attempt to explore the follow directions of development:

- Extend the two-sided model in Fig. 2 into more realistic Internet topologies, for example include transit ISPs in the model.

- Compare the Shapley value solution with the current bilateral agreements among ISPs and explore its regulatory implications on future evolutions of the ISP settlements.

- Seek bilateral implementations of the Shapley value solution.

We believe that the Shapley value solution will provide guidelines for solving disputes between ISPs and for establishing regulatory protocols for the industry.

\section{REFERENCES}

[1] A. Roth, The Shapley value: Essays in honor of Lloyd S. Shapley. Cambridge University Press, Cambridge, 1988.

[2] E. Winter, The Shapley Value, in The Handbook of Game Theory. R. J. Aumann and S. Hart, North-Holland, 2002.

[3] R. T. B. Ma, D. Chiu, J. C. Lui, V. Misra, and D. Rubenstein, "Internet Economics: The use of Shapley value for ISP settlement," Proceedings of 2007 ACM Conference on Emerging network experiment and technology (CoNEXT 2007), December 2007.

[4] _ "Interconneting Eyeballs to Content: A Shapley Value Perspective on ISP Peering and Settlement," Proceedings of 2008 ACM Network Economics (NetEcon), August 2008.

[5] R. Myerson, "Graphs and cooperation in games," Mathematics of Operations Research, vol. 2, pp. 225-229, 1977.

[6] P. Faratin, D. Clark, P. Gilmore, S. Bauer, A. Berger, and W. Lehr, "Complexity of Internet interconnections: Technology, incentives and implications for policy," The 35th Research Conference on Communication, Information and Internet Policy (TPRC), 2007. 